\title{
A Three Year Retrospective Study on Seroprevalence of Syphilis among Pregnant Women at Gondar University Teaching Hospital, Ethiopia.
}

\author{
Abate Assefa \\ University of Gondar, College of Medicine and Health Sciences, School of Biomedical and Laboratory Sciences, Department \\ of Medical Microbiology
}

\begin{abstract}
Background: Sexually transmitted infections (STIs) are a serious public health problem in low income countries, including Ethiopia. Syphilis caused by Treponema pallidum remains a major cause of reproductive morbidity and poor pregnancy outcomes in low income countries. Stillbirth, perinatal death, serious neonatal infection and low-birth weight babies are common among seropositive mothers.

Objective: To assess the seroprevalence of syphilis and risk factor correlates of this infection at Gondar University Teaching Hospital, Ethiopia.

Methods: The study was done on 2385 pregnant women attending the antenatal care clinic (ANC) from January 2009 to December 2011. Data was abstracted from the antenatal care clinic medical database. Chi-square test was used, using SPSS version 16 and significance level was chosen at 0.05 level with a two-tailed test.

Results: Of the total, 69(2. 9\%) of pregnant women were confirmed as seropositive for syphilis. Pregnant women with an age group of 21-25 years of old were the most seropositive (3.4\%), followed by $26-30$ years of old (3.1\%). The prevalence of syphilis infection was $3.2 \%$ in urban and $2.2 \%$ in rural pregnant women. Relatively high prevalence of syphilis infection were identified among students $(4.2 \%)$ followed by governmental employees $(3.8 \%)$.

Conclusion: The seroprevalence of syphilis among pregnant women attending ANC is declining. However, syphilis is more prevalent in the young and urban pregnant women. Emphasis on education to young people on STI risk behavioral change and partner follow up and notification for exposure to syphilis and treatment should be given.
\end{abstract}

Key word: Pregnant women, seroprevalence, syphilis

African Health Sciences 2014;14(1): 119-124 http:/ /dx.doi.org/10.4314/ahs.v14i1.18

\section{Introduction}

Sexually transmitted infections (STIs) are a serious public health problem in developing countries, including Ethiopia (1). Syphilis is STIs caused by the spirochete Treponema pallidum and constitutes a major public health problem worldwide (2). Syphilis is an important cause of morbidity and mortality in pregnancy and it is one of the greatest public health challenges (3). Pregnant women are sexually active and at risk of sexually transmitted disease, including syphilis. Syphilis remains a major cause of reproductive morbidity and poor pregnancy outcomes in developing countries. In prenatal care

\section{Corresponding author:}

Abate Assefa

University of Gondar, College of Medicine and Health Sciences, School of Biomedical and Laboratory Sciences

Department of Medical Microbiology

E-mail address: abazew@gmail.com screening and treatment for syphilis is routine and is a cost-effective intervention (4). Fetal infection is a result of hematogeneous spread from an infected mother. Even though Treponema pallidum can cross the placenta and infect the fetus in the early gestation, the risk of adverse pregnancy outcomes increases with age. Adverse pregnancy outcomes are influenced by gestational age, stage of maternal syphilis, and immunological response of the fetus. In the early gestational age, the fetus is not capable of consistently mounting an immune response to the infection which is a primary factor for the development of syphilis infection clinical outcomes (5).

Beside many African adults who die from syphilis each year, babies born to syphilitic mothers often contract congenital syphilis and suffer from horrible symptoms associated with it. According to 2007 world health organization (WHO) report syphilis infection rates in pregnant women in Africa as a whole ranges from 3 to15\% (6). Untreated syphilis in pregnancy leads to adverse outcomes among more than half of the women with active disease. If syphilis in pregnant women is not 
treated, her fetus is negatively affected in almost $80 \%$ of cases, resulting into $40 \%$ stillbirth, $20 \%$ perinatal death and serious neonatal infection and 20\% low-birth weight babies (4).

In sub-Saharan Africa estimated that in pregnancy active syphilis infection can cause adverse outcomes in 50-80\% of pregnancies surviving post 12 weeks conception and syphilis sero-reactivity ranges from $4-15 \%$ (7). Recent data from Malawi and Tanzania indicated that $21 \%$ of perinatal deaths, $26 \%-51 \%$ of stillbirths, $24 \%$ of preterm live births, $17 \%$ of all adverse pregnancy outcomes and $11 \%$ of neonatal deaths have been attributed to untreated seropositive pregnant mothers (8). Further more, in Ethiopia studies have demonstrated that $21 \%$ children born from seropositive mothers had developed signs of syphilis. Stillbirth and abortion rates of infected women were almost double than among the general population (9). Therefore, for better understand the syphilis epidemiology among pregnant women in Ethiopia, the seroprevalence of syphilis and sociodemographic correlates of this infection at Gondar university teaching hospital, Ethiopia, was assessed.

\section{Method and materials}

\section{Study design and subjects}

A retrospective study was carried out on 2385 pregnant women attending at Antenatal Care Clinic (ANC) of Gondar university hospital from January 2009 and December 2011. Gondar university hospital is a referral hospital with more than 400 beds for Northwest Ethiopia serving a population of about 5 million. All pregnant women attending ANC of Gondar university hospital were eligible for the study. Antenatal care services for all pregnant women provide routine laboratory diagnostic tests. Serology test for syphilis is one of the important tests to be done during the first ANC visit. In serology laboratory of the hospital a $5 \mathrm{ml}$ of venous blood was collected from the antecubital vein of each woman into sterile tubes. The blood was allowed to retract and then centrifuged, and the serum was obtained. All serum samples, test antigens and control samples were brought to room temperature and tested by rapid plasma reagin (RPR) test. All RPR positive sera were subjected to the Treponema pallidum haemagglutination (TPHA) test as a confirmatory test. When RPR and TPHA were positive then patient has syphilis.

\section{Data collection}

The study was reviewed and approved by ethical review committee of the school of biomedical and laboratory sciences, University of Gondar. The extracted data for the study were analysed anonymously. Individual records also coded and accessed only by research staff. Data on demographic parameters, age, residence and occupation, were abstracted from antenatal care clinical records. Laboratory data of seropositivity of syphilis were obtained from Gondar university hospital serology laboratory. Pregnant women whose charts were unavailable for reviews were excluded from the analysis.

\section{Statistical Analysis}

Data entry and analysis was done using SPSS version-16 software. A descriptive analysis was used to determine socio-demographic characteristics and seroprevalence of syphilis. The differences in proportions of syphilis for associated risk factors were tested by the chi-square $\left(\chi^{2}\right)$ test. Significance levels were chosen at 0.05 level with a two-tailed test.

\section{Result \\ Demographic characteristics}

A total of 2385 pregnant women were examined for seropositivity of syphilis between January 2009 and December 2011. The age of the women ranged from 16-45 years with a mean of 30.5 years. The majority of women were in the 21-25 years age group (34.9\%). One thousand and seven hundred forty five $(73.2 \%)$ pregnant women were living in urban and most of the women were housewives $(77.7 \%)$. In terms of years of study period, the highest numbers of ANC attendees were 1021(42.8\%) obtained in the year 2009 (table1). 
Table 1. The socio-demographic characteristics of pregnant women attending Gondar university hospital

\begin{tabular}{llll}
\hline Characteristic & Number & Percentage \\
\hline Age in year & $16-20$ & 536 & 22.5 \\
& $21-25$ & 823 & 34.5 \\
& $26-30$ & 673 & 28.2 \\
& $31-35$ & 237 & 9.97 \\
& $36-40$ & 93 & 3.89 \\
Residence & Ru-45 & 23 & 0.96 \\
& Rural & 640 & 26.8 \\
& Urban & 1745 & 73.2 \\
& Housewife & 1654 & 77.7 \\
& Government Employee & 212 & 8.9 \\
& Students & 125 & 5.2 \\
& Teachers & 110 & 4.6 \\
& Health Professional & 30 & 1.3 \\
& Daily worker & 28 & 1.2 \\
& Others & 26 & 1.1 \\
& 2009 & 1021 & 42.8 \\
& 2010 & 722 & 30.3 \\
& 2011 & 642 & 26.9
\end{tabular}

Syphilis seroprevalence and associated risk factors Of the total pregnant women, $69(2.9 \%)$ were confirmed as seropositive for syphilis. Relatively high seroprevalence of syphilis were observed among age groups of $21-25$ years of old (3.4\%) followed by in the age group of 26-30 years of old (3.1\%). Even though there was a difference in the magnitude of seropositivity of syphilis within different age groups, the difference was not statistically significant $\left(\chi^{2}=4.36, p=0.43\right)$. The difference in proportion of seropositivity between urban $(3.2 \%)$ and rural $(2.2 \%)$ dwellers of pregnant women was also not statistically significant $\left(\chi^{2}=1.54\right.$, $\mathrm{p}=0.21)$. In terms of occupation, students $(4.2 \%)$ and governmental employee $(3.8 \%)$ were more affected than other categories of occupations. However, the difference in the distribution of syphilis among the various occupations was no statistically significant (table 2). 
Table 2. The seroprevalence of syphilis and associated risk factors among pregnant women attending Gondar university hospital

\begin{tabular}{|c|c|c|c|c|c|c|}
\hline \multirow[t]{2}{*}{ Characteristic } & \multirow[t]{2}{*}{$\mathrm{n}(\%)$} & \multicolumn{3}{|c|}{ Syphilis seroreactivity } & \multirow[b]{2}{*}{$\chi^{2} n(\%)$} & \multirow[b]{2}{*}{ P-value } \\
\hline & & & $\begin{array}{l}\text { Yes } \\
\mathrm{n}(\%)\end{array}$ & No & & \\
\hline \multirow[t]{6}{*}{ Age (in year } & $16-20)$ & $536(22.5)$ & $16(2.5)$ & $520(97.0)$ & & \\
\hline & $21-25$ & $823(34.5)$ & $28(3.4)$ & $795(96.6)$ & & \\
\hline & $26-30$ & $673(28.2)$ & $21(3.1)$ & $652(96.9)$ & 4.4 & 0.43 \\
\hline & $31-35$ & $237(9.9)$ & $3(1.3)$ & $234(98.7)$ & & \\
\hline & $36-40$ & $93(3.9)$ & $1(1.1)$ & $92(98.9)$ & & \\
\hline & $41-45$ & $23(1.0)$ & $0(00)$ & $23(100)$ & & \\
\hline \multirow[t]{2}{*}{ Residence } & Rural & $640(26.8)$ & $14(2.2)$ & $626(97.8)$ & & \\
\hline & Urban & $1745(73.2)$ & $55(3.2)$ & $1690(96.8)$ & 1.5 & 0.21 \\
\hline \multirow[t]{7}{*}{ Occupation } & Housewife & $1654(77.7)$ & $49(2.6)$ & $1805(98.4)$ & & \\
\hline & Employed & $212(8.9)$ & $8(3.8)$ & $204(96.2)$ & & \\
\hline & Students & $125(5.2)$ & $5(4.2)$ & $120(95.8)$ & & \\
\hline & Teachers & $110(4.6)$ & $4(3.6)$ & $106(96.4)$ & 1.8 & 0.9 \\
\hline & Health Professional & $30(1.3)$ & $1(3.3)$ & $29(96.7)$ & & \\
\hline & Daily worker & $28(1.2)$ & $1(3.6)$ & $27(96.4)$ & & \\
\hline & Others & $26(1.1)$ & $1(3.8)$ & $25(96.2)$ & & \\
\hline \multirow[t]{3}{*}{ Study year } & 2009 & $1021(42.8)$ & $30(2.9)$ & $991(97.1)$ & & \\
\hline & 2010 & $722(30.3)$ & $14(1.9)$ & $708(98.1)$ & 4.6 & 0.09 \\
\hline & 2011 & $642(26.9)$ & $25(3.9)$ & $617(96.1)$ & & \\
\hline
\end{tabular}

\section{Discussion}

The prevalence of syphilis in this study was $2.9 \%$. The majority of ANC attendees came from urban areas. This may be due to problems of accessibility and less awareness of the services by the rural population. Among women who attend at ANC of Gondar university hospital, in Ethiopia, the seroreactivity was a little bit high in the year of 2009. Yet, in 2010 seroprevalence of syphilis showed decreasing, again in 2011, an increased seroprevalence was observed. The overall prevalence of syphilis is consistent with the result reported from Addis Ababa 2.9\% (10). This figure is slightly higher than the seroprevalence of syphilis reported from Burkina Faso $(0.24 \%)$ (11), Nigeria $(0.07 \%)$ (12) and rural China $(0.39 \%)(13)$. However, this result is lower than previous reports from Gondar health center in 2006 (23\%) (14), Debretabor (13.7\%) (15), Jimma hospital $(12.1 \%)(16)$, urban and semi-urban health centers of Ethiopia (8.6\%) (17), sub-Saharan African country (8.3\%) (7), Democratic Republic of Congo (4.2\%) (18) and Uganda $(4.0 \%)(19)$. These figures may indicate as there has been significant change in the prevalence

of syphilis in Ethiopia over the last decade. The low rate of syphilis in the study area may be due to safer sexual practice and antibiotic use as well as increasing of appropriate STI service delivery.

In this study we observed a relatively an age-related downward trend of seroprevalence of syphilis. The seroprevalence of syphilis decreased with increasing age. Women with an age group of 21-25 years of old were the most seropositive (3.4\%), followed by the age group of 26-30 years of old (3.1\%). Nevertheless, women at age group of 36 years old and above were the least vulnerable to syphilis. These findings were concordant with the report in Nigeria (12). In contrast, the report from Jimma revealed that the most affected age groups were 15-19 years old (16). The variations of syphilis prevalence with age may be explained as a reflection of the differences in sexual practices, such as number of sexual partners. Hence, these findings highlighted that the younger age groups are relatively prone to syphilis infection. This may be due to unsafe sexual practices like unprotected sex. 
Although the relation is not significant, pregnant women living in urban areas had a higher chance of being seropositive for syphilis than rural dwellers. In contrast to our findings significantly higher seropositivity of syphilis among pregnant women attending rural clinics $(5.0 \%)$ as compared to urban clinics $(3.0 \%)$ was reported from Democratic Republic of Congo (18). Our findings of $3.2 \%$ seropositivity of syphilis in urban pregnant women population was much lower than the previous study reported in Ethiopia from urban and semi-urban health centers, which was $13.3 \%$ and most seropositive pregnant women were rural dwellers (31.2\%) (17). Similarly, the report from Debretabor rural hospital showed that seropositivity of syphilis $(13.7 \%)$ was much higher than our finding $(2.2 \%)$ in rural pregnant women population (15). This difference may be due to lack of awareness about STIs in rural areas. However, in this study, none of these factors were found to be statistically associated with prevalence of syphilis. This finding is in line with the report of Kebede et al from Addis Ababa (10). Unidentified environmental or host factors might be the prime determinants of the seroprevalence distribution in the current study subjects. Though the difference of proportion of seropositivity of syphilis among various occupations of women was not statistically significant, in the current study least affected groups were housewife and health professional with $2.4 \%$ and $3.3 \%$ respectively.

\section{Conclusion}

In conclusion, the study indicated that the seroprevalence of syphilis among pregnant women attending ANC is declining. However, relatively syphilis is more prevalent in the young ( $21-25$ years old) and urban pregnant women. Emphasis on education to young people on STI risk behavioral change and partner follow up and notification for exposure to syphilis and treatment should be given.

\section{Declaration of competing interests}

The author declares that he has no competing interests.

\section{References}

1. World Health Organization (WHO). Guideline for the Management of Sexually Transmitted Infections. Revised version. WHO, Geneva, Switzerland; 2003. Avaliable at: http://www.who.int/hiv/pub/sti/en/ STIGuidelines2003.pdf.
2. Nakashima AK, Rolfs RT, Flock ML, Kilmarx P, Greenspan JR. Epidemiology of syphilis in the United States, 1941-1993. Sex Transm Dis 1996; 23(1): 16-23.

3. Schmid G. Economic and programmatic aspects of congenital syphilis prevention. Bull World Health Organ 2004; 82(6):402-409.

4. Mullick S, Watson-Jones D, Beksinska M, Mabey D. Sexually transmitted infections in pregnancy: prevalence, impact on pregnancy outcomes, and approach to treatment in developing countries. Sex Transm Infect 2005; 81:294-302.

5. Saloojee H, Velaphi S, Goga Y, Afadapa N, Steen $\mathrm{R}$, Lincetto $\mathrm{O}$. "The prevention and management of congenital syphilis: an overview and recommendations". Bull World Health Organ 2004; 82(6):424-430.

6. World Health Organization (WHO). The Global Elimination of Congenital Syphilis: Rationale and Strategy for Action. WHO, Geneva, Switzerland; 2007. Available at: http://whqlibdoc.who.int/ publications/2007/9789241595858_eng.pdf.

7. Gloyd S, Chai S, Mercer MA. Antenatal syphilis in sub -Saharan Africa: missed opportunities for mortality reduction. Health Policy Plan 2001; 16(1): 29-34.

8. Centers for Disease Control and Prevention. Syphilis control during pregnancy: Effectiveness and sustainability of a decentralized program. $M M W R$. 2001; 51:853-856.

9. Naeye RL, Tafari N, Marboe CC, Judge DM. Causes of perinatal mortality in an African city. Bull World Health Organ 1977; 55(1):63-65.

10. Kebede E. Chamiso B. Prevalence of syphilis in pregnancy in Addis Ababa. East Afr Med J 2000; 77 (4): 212-216.

11. Issiaka S, Nicolas M, Michel C, Sylvestre T, Amadou O, Seydou Y, et al. "Seroprevalence of Syphilis among Women Attending Urban Antenatal Clinics in Burkina Faso, 1995-1998." Sex Trans infect 2000; 76(4): 314-316. 12. Bukor M, Audu BM, Takoivi A, joy BB, killimo A. is routine antenatal screening for syphilis in Nigeria still justified clinically and economically? Saudy med J 2009; 30(10): 1311-1315.

13. Yang LG, Tucker JD, Liu FY, Ren XQ, Hong X, , Wang C, et al. O Syphilis Screening among 27,150 Pregnant Women in South Chinese Rural Areas Using Point-of-Care Tests. PLoS ONE 2013; 8(8): e72149.

14. Trunech M. Sero- prevalence of multiple sexually transmitted infections among ANC attended in Gondar Health center North West Ethiopia. Ethio Med J 2008; 46(4): $359-366$.

15. Kidan KG, Fantahun M, Azeze B. Seroprevalence 
of Human Immunodeficiency Virus Infection and Its Association with Syphilis Seropositivity among Antenatal Clinic Attenders at Debretabor Referral Hospital, Ethiopia. East Afr Med J 1995; 72(9):579_ 585.

16. Mekonnen Z, Tegbaru B, Meless, H. Sero- prevalence of syphilis and HIV-1 among pregnant women attending ANC clinic in Jimma Hospital South Western Ethiopia. Ethio J health Sciences 2002; 12(2): 81-89.

17. Felke W, Yusuf M, Teshy H, Abraham A, Carlosi G. Prevalence of syphilis among pregnant women attending urban and semi urban health centers in Ethiopia. STD, AIDS control program in Ethiopia. Intl
Conf AIDS1992; 8(2):B148.

18. Taylor MM, Ebrahim S, Abiola N, Kinkodi DK, Mpingulu M, Kabuayi JP, et al.Correlates of syphilis seropositivity and risk for syphilis-associated adverse pregnancy outcomes among women attending antenatal care clinics in the Democratic Republic of Congo. Int J STD AIDS 2014; [article in press].

19. Kizito D, Woodburn PW, Kesande B, Ameke C, Nabulime J, Muwanga M, et al. Uptake of HIV and syphilis testing of pregnant women and their male partners in a programme for prevention of mother-tochild HIV transmission in Uganda. Trop Med Int Health 2008; 13(5):680-682. 